\title{
HIGLY-RESOLVED NUMERICAL AND LABORATORY ANALYSIS FOR NONBREAKING SOLITARY WAVE SWASH OVER A STEEP SLOPE
}

\author{
Pablo Higuera, National University of Singapore, pablo.higuera@nus.edu.sg \\ Philip L.-F. Liu, National University of Singapore and Cornell University, philip.liu@nus.edu.sg \\ Cheng Lin, National Chung Hsing University, chenglin@nchu.edu.tw \\ Wei-Ying Wong, National Chung Hsing University, wongweiying@gmail.com \\ Ming-Jer Kao, National Chung Hsing University, d9362202@mail.nchu.edu.tw
}

\section{INTRODUCTION}

In this paper we study the swash processes generated by a nonbreaking solitary wave running up and down a steep slope (1:3). We use experimental data to study flow features and velocities inside the boundary layer, and numerical modelling to investigate variables not measured during the laboratory experiments, such as pressures and bottom shear stress. We focus on the mechanisms that produce flow separation and vortex formation. Particularly, we study a system of vortices generated under a hydraulic jump during the rundown phase, which was first observed by Matsunaga \& Honji (1980).

\section{PHYSICAL EXPERIMENTS}

Physical experiments (Lin et al., 2015) were performed at the National Chung Hsing University in a $14 \mathrm{~m}$ long flume, with a still water depth of $h=8 \mathrm{~cm}$. Solitary waves $(\mathrm{H} / \mathrm{h}=0.363)$ were generated with a piston-type wavemaker. A steep slope (1:3) was installed at the other edge of the flume. A high-speed particle image velocimetry (HSPIV) system captured high resolution free surface elevation and velocity measurements at different scales and locations along the flume, including inside the bottom boundary layer. When operated at lower shutter speeds, the system also serves for flow feature visualization, in which the vortices can be distinguished and the features measured, as shown in Figure 1. The runup height was also monitored. The experimental data provides comprehensive information of the different phases that the flow undergoes, comprising wave shoaling, runup, rundown, hydraulic jump and wave breaking.

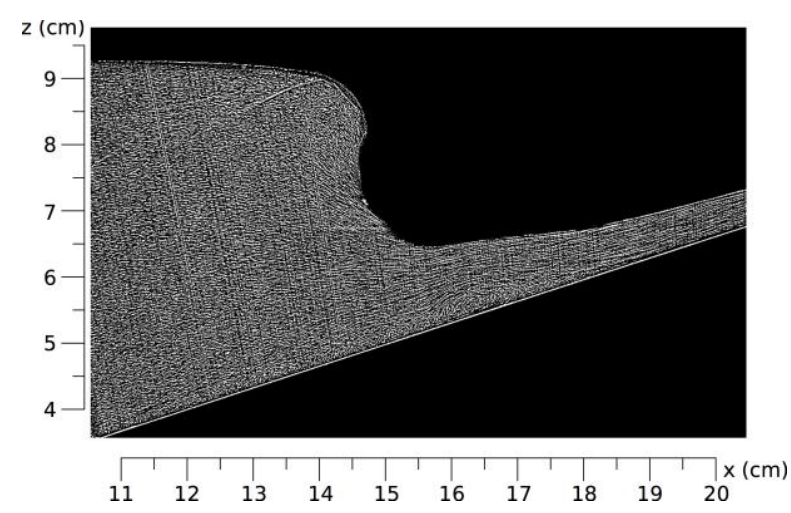

Figure 1 - Flow separation visualization by the HSPIV system during the hydraulic jump formation in the rundown phase. The main vortex in the separation region is located at $\mathrm{x}=16.25 \mathrm{~cm}$.

\section{NUMERICAL EXPERIMENTS}

The physical experiments have been replicated with olaFlow, an enhanced version of the 3D ReynoldsAveraged Navier-Stokes numerical model based on the OpenFOAM library presented in Higuera et al. (2013). The numerical model is used to extend the experimental database, after a careful convergence analysis and validation against the experimental data. The numerical results overcome some experimental limitations and provide highly-resolved data in time and space for velocity and free surface elevation, as well as for additional variables (e.g. pressure gradients, bottom shear stress).

The numerical mesh (8.8 million cells) is unstructured and replicates a shorter portion of the wave flume in 3D. The simulation takes advantage of the symmetrical setup, simulating a shorter flume and half of the domain to save computational resources. The general cell resolution is $1 \mathrm{~mm}$ in the wave propagation and vertical directions, and $2 \mathrm{~mm}$ in the spanwise direction. The resolution near the bottom has been enhanced to 0.1 $\mathrm{mm}$ in the vertical direction, to capture the boundary layer structure in great detail. Turbulence has been modeled with the k-omega SST model presented in Devolder et al. (2017), which suppresses the spurious turbulence generation at the free surface, preventing turbulence buildup. The simulation is $4.5 \mathrm{~s}$ long and takes 130 hours running in parallel in a Xeon workstation (24 cores, $2.50 \mathrm{GHz}$ ).

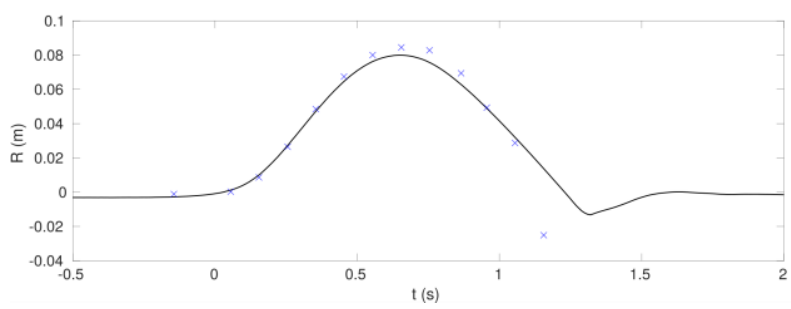

Figure 2 - Time series of runup height. Numerical data (line) and experimental measurements (crosses).

\section{RESULTS}

The runup curve measured in the numerical simulation is compared against the experimental data points in Figure 2. The numerical curve does not start at the zero level because a meniscus develops at the interface between water and the slope, causing a retreat of the shoreline. The degree of agreement during the initial runup phase 
is adequate. Differences increase with time due to error accumulation, caused by inaccuracies in the numerical modelling of the shoreline dynamics. Nevertheless, errors are local to the moving shoreline and the general agreement of velocity profiles is excellent elsewhere, including inside the bottom boundary layer. The maximum runup height in the model is slightly underestimated (4 mm, $4.5 \%)$.
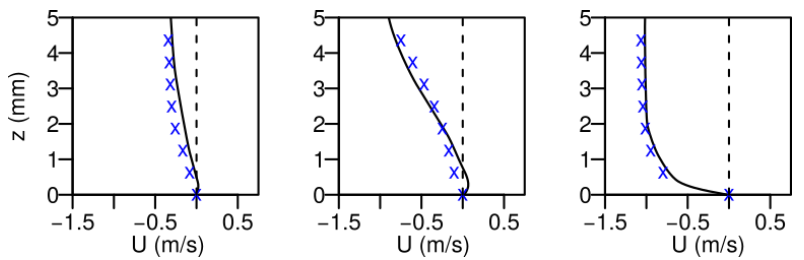

Figure 3 - Horizontal velocity inside the boundary layer at $t$ $=1.01 \mathrm{~s}$. From left to right: $\mathrm{x}=12.36 \mathrm{~cm}, \mathrm{x}=15.49 \mathrm{~cm}$ and $x=18.62 \mathrm{~cm}$. Numerical data (line) and experimental measurements (crosses).

Although the deviations in runup height increase during the rundown phase, introducing minor time and space lags produce an adequate matching of velocities during the entire rundown phase. Lags are calculated to line up the hydraulic jump front in the numerical simulation and in the experiments.

Figure 3 shows velocity profile comparisons at three locations on the slope for an instant of the rundown phase. Both the velocity inside the boundary layer and in the free-stream region are well replicated by the numerical model.

Flow separation and vortex shedding occur during rundown near the hydraulic jump, in an area in which the flow decelerates heavily, transitioning from supercritical to subcritical regime (Figure 1). The pressure gradient induced by the sharp difference in free surface elevation is large enough to overcome the momentum of the downwash flow, producing flow reversal starting from the bottom, as in the central panel in Figure 3.

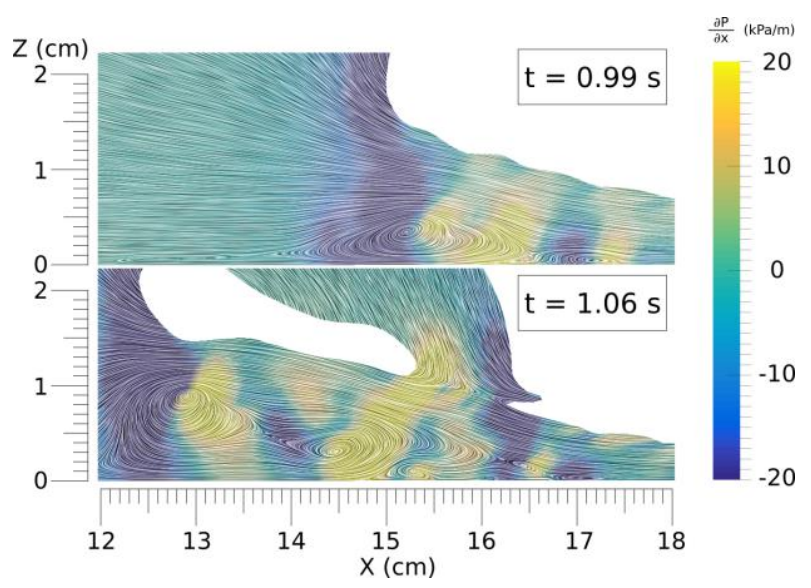

Figure 4 - LIC visualization of the vortex system and pressure gradient in the vicinity of the hydraulic jump.

Figure 4 shows the Line Integral Convolution visualization of the vortex system generated in a $2 \mathrm{D}$ slice of the numerical domain at two instants: while the hydraulic jump is overturning and at wave impingement. The pressure gradient values in the horizontal direction (free from gravity effects) have been superimposed and point out that vortices are linked to sharp variations in the pressure gradient, from negative to positive in the onshore to offshore direction. The evolution of the system starts with a very small vortex, that grows larger as time advances. This initial vortex rotates in the counter-clockwise direction and is advected down the slope by the downwash flow. As it moves downstream other counter-clockwise vortices are generated upstream, near it (Figure 4, top panel). This pair produces a small clockwise vortex in the middle of them.

The numerical results of bottom shear stress (BSS) agree well with previous studies. The maximum BSS takes place at the shoreline both for the runup and rundown phases, as observed in Pujara et al. (2015). Moreover, the magnitude of the maximum BSS during runup is approximately double than that during rundown. The evolution of the BSS also resembles that described in Sumer et al. (2011) [Figure 9 a \& b], although the slope was milder in their case and produced wave breaking before runup.

\section{CONCLUSIONS}

The combined use of experimental and numerical results has helped us gain a better understanding of the physical processes encompassed in the swash of a nonbreaking solitary wave, qualitatively and quantitatively. The three-dimensional simulation has proven useful to provide highly-resolved values in time and space for variables such as pressure and bottom shear stress, not measured in the experiments. Additional materials, as Froude number, will be included and discussed in the presentation.

\section{REFERENCES}

Devolder, Rauwoens \& Troch (2017): Application of a buoyancy-modified k-omega SST turbulence model to simulate wave run-up around a monopile subjected to regular waves using OpenFOAM. Coastal Engineering, vol. 125, pp. 81-94.

Higuera, Lara \& Losada (2013): Realistic wave generation and active wave absorption for Navier-Stokes models: Application to OpenFOAM. Coastal Engineering vol. 71, pp. 102-118.

Lin, Yeh, Kao, Yu, Hseih, Chang, Wu \&Tsai (2015): Velocity fields in near-bottom and boundary layer flows in pre-breaking zone of solitary wave propagating over a 1:10 slope. Journal of Waterways, Port, Coastal, and Ocean Engineering, vol. 141(3), pp. 1-30.

Matsunaga \& Honji (1980): The backwash vortex. Journal of Fluid Mechanics, vol. 99(4), pp. 813-815.

Pujara, Liu \& Yeh (2015): The swash of solitary waves on a plane beach: flow evolution, bed shear stress and runup. Journal of Fluid Mechanics, vol. 779, pp. 556-597.

Sumer, Sen, Karagali, Ceren, Fredsoe, Sottile, Zilioli \& Fuhrman (2011): Flow and sediment transport induced by a plunging solitary wave. Journal of Geophysical Research, vol. 116, C01008. 